Research Paper

\title{
Multiple-, But Not Single-, Dose of Parecoxib Reduces Shoulder Pain after Gynecologic Laparoscopy
}

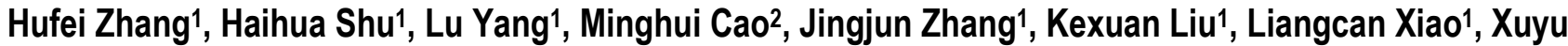 \\ Zhang $^{1 凶}$
}

1. Department of Anesthesiology, The First Affiliated Hospital, Sun Yat-sen University, Guangzhou, China;

2. Department of Gynecologic Laparoscopic Medical Center, The First Affiliated Hospital, Sun Yat-sen University, Guangzhou, China

$\triangle$ Corresponding author: Xuyu Zhang, M.D., Department of Anesthesiology, The First Affiliated Hospital, Sun Yat-sen University, No.58, Zhongshan 2nd Road,Guangzhou, P. R. China. Phone: 0086-020-87755766-8273. FAX: 0086-020-87755766-8271. Email: Liuzm@sysu.edu.cn

(C) Ivyspring International Publisher. This is an open-access article distributed under the terms of the Creative Commons License (http://creativecommons.org/ licenses/by-nc-nd/3.0/). Reproduction is permitted for personal, noncommercial use, provided that the article is in whole, unmodified, and properly cited.

Received: 2012.07.23; Accepted: 2012.10.17; Published: 2012.10.23

\begin{abstract}
Background: The aim of this study was to investigate effect of single- and multiple-dose of parecoxib on shoulder pain after gynecologic laparoscopy.

Methods: 126 patients requiring elective gynecologic laparoscopy were randomly allocated to three groups. Group M (multiple-dose): receiving parecoxib $40 \mathrm{mg}$ at $30 \mathrm{~min}$ before the end of surgery, at 8 and $20 \mathrm{hr}$ after surgery, respectively; Group S (single-dose): receiving parecoxib $40 \mathrm{mg}$ at $30 \mathrm{~min}$ before the end of surgery and normal saline at the corresponding time points; Group C (control): receiving normal saline at the same three time points. The shoulder pain was evaluated, both at rest and with motion, at postoperative 6,24 and 48hr. The impact of shoulder pain on patients' recovery (activity, mood, walking and sleep) was also evaluated. Meanwhile, rescue analgesics and complications were recorded.

Results: The overall incidence of shoulder pain in group $M(37.5 \%)$ was lower than that in group C (6I.9\%) (difference=-24.4\%; 95\% Cl: 3.4 45.4\%; $\mathrm{P}=0.023$ ). Whereas, single-dose regimen $(6 \mathrm{I} .0 \%$ ) showed no significant reduction (difference with control $=-0.9 \% ; 95 \% \mathrm{Cl}$ : $-21.9 \sim 20.0 \% ; P=0.931$ ). Moreover, multiple-dose regimen reduced the maximal intensity of shoulder pain and the impact for activity and mood in comparison to the control. Multiple-dose of parecoxib decreased the consumption of rescue analgesics. The complications were similar among all groups and no severe complications were observed.

Conclusions: Multiple-, but not single-, dose of parecoxib may attenuate the incidence and intensity of shoulder pain and thereby improve patients' quality of recovery following gynecologic laparoscopy.
\end{abstract}

Key words: laparoscopic surgery, Shoulder pain, postoperative pain, nonsteroidal anti-inflammatory drugs

\section{Introduction}

Laparoscopic procedures, compared to laparotomies, result in smaller incision, lower morbidity, quicker recovery and less postoperative pain [1-3]. Therefore, laparoscopic surgery has revolutionized the practice of many surgical subspecialties over the past 2 decades [4]. However, laparoscopic procedures are often associated with postlaparoscopic shoulder pain (PLSP), which may cause more discomfort to the patients than the pain at the incision sites [5]. The reported incidence of PLSP was $63 \%$ after laparoscopic 
cholecystectomy [6], 66\% after laparoscopic adjustable gastric band surgery [7], 65.5\% after laparoscopic appendectomy ${ }^{[8]}$ and 83\% after gynecological laparoscopic surgeries [5]. For improving the postoperative quality of life (QOL) for these patients, various techniques, including low-pressure insufflation, no $\mathrm{CO}_{2}$ insufflation, preemptive diaphragmatic local anesthetic irrigation and regional anesthesia to peritoneal surfaces, have been developed to reduce the PLSP [9-13]. Unfortunately, the effectiveness of these techniques was quite varied and even conflicting [7]. Moreover, many of these techniques were complicated and difficult for clinical application [5].

Because the exact mechanism of PLSP remains unclear [5,8], it is difficult to choose an optimal therapeutic drug. Nonsteroidal anti-inflammatory drugs (NSAIDs) have been widely applied for postoperative pain management after laparoscopic surgery [14, 15]. Several trials showed that NSAIDs, either selective or non-selective, were not superior to placebo on shoulder pain control although they could reduce surgical pain or rescue narcotics requirement [16-19]. Alanoglu et al. reported that preoperative use of celecoxib or rofecoxib decreased shoulder pain score only within postoperative $1 \mathrm{hr}$ after laparoscopic Nissen fundoplication [15], and Jabbour-Khoury et al. indicated that intravenous ketoprofen just provided analgesic effect on shoulder pain during 6hrs in laparoscopic cholecystectomy [20]. However, a study showed that preemptive celecoxib in day-case diagnostic laparoscopy reduced PLSP within postoperative 24hrs but had no advantage of decreasing wound pain and the total analgesic requirements [21]. So far, the analgesic effect of NSAIDs on PLSP is controversial. Parecoxib, a cyclooxygenase-2 (COX-2) selective inhibitor, has an efficient and rapid ability to cross the blood-brain barrier ${ }^{[22,23]}$ and possesses better peripheral and central antihyperalgesic effects [24]. The effectiveness of parecoxib for postoperative shoulder pain in laparoscopy is not clear. Meanwhile, because single-dose application of NSAIDs was applied, either before or during operation, in all previous studies associated with PLSP, a multiple-dose regimen was designed to find an appropriate analgesic regimen for shoulder pain. We hypothesized that the suitable application of parecoxib might be the effective treatment for PLSP.

The aim of this study was to investigate the effect of parecoxib, administered through single- or multiple-dose regimen, on shoulder pain following gynecologic laparoscopy.

\section{Methods}

After institutional review board of $1^{\text {st }}$ affiliated hospital of Sun Yat-sen university approval and written informed consent obtained from the patients, age18-65yr, American Society of Anesthesiology I-II female patients undergoing elective gynecologic laparoscopic surgery with general anesthesia were recruited for this randomized, double-blind and placebo controlled study to assess whether multiple- or singledose regimen of parecoxib could reduce shoulder pain occurrence when compared to the control. Exclusion criteria were: 1 ) Body Mass Index(BMI) $<18$ or $>25 \mathrm{~kg} / \mathrm{m}^{2} ; 2$ ) patients with a history of severe cardiac, pulmonary, hepatic, renal disease, chronic drug or alcohol abuse; 3) the presence of preoperative shoulder pain or any chronic pain syndrome, long-term use of analgesics; 4) allergy or contraindications to NSAIDs and any other drugs used in the study; 5) Patients with psychological or other disorders that pain evaluation was difficult to be judged. In addition, patients with conversion to a laparotomy and with operation time less than $0.5 \mathrm{hr}$ were eliminated from the study.

Patients who met the inclusion criteria were randomly assigned by the study physician to one of three groups from a pre-assigned list, which was computer generated by using SAS software. Randomization lists were assigned in blocks of six, each comprising a list of the same six letters in random order. The three groups were each assigned two randomly selected letters from among the six, which were used to label the packaging of the drug. Both the randomization lists and envelops were stored securely by an independent technician. Participants, care providers and research team were masked to group assignment.

Study drugs were distributed in sealed opaque packs, identified only by drug group and stored in an envelope. On the day of surgery, an attending doctor who was not involved in anesthetic management and postoperative assessment acquired the next envelope in the sequence and opened it at the start of surgery. This doctor independently prepared and infused the drugs according to grouping during the whole study period. The patients were randomly allocated into three groups. Group M (multiple administrations of parecoxib): parecoxib $40 \mathrm{mg}$ dissolved in $5 \mathrm{ml}$ normal saline (NS) was intravenously administrated at $30 \mathrm{~min}$ before the end of surgery, at $8 \mathrm{hr}$ and $20 \mathrm{hr}$ after the surgery, respectively; Group S (single administration of parecoxib): the patients received parecoxib $40 \mathrm{mg}$ intravenously $30 \mathrm{~min}$ before the end of the surgery, followed by NS $5 \mathrm{ml}$ intravenously at indicated time after surgery; Group C (control): NS $5 \mathrm{ml}$ was intravenously administrated at above time points, respectively. The time of parecoxib administration was designed based on the pharmacodynamics of parecoxib 
(Dynastat, Pharmacia Limited) and the rest time of patients.

After admission to the operation room, vital signs and Narcotrend Index were monitored. All patients were managed by a propofol-based general anesthesia with a fixed continuous intravenous remifentanil at $0.25 \mu \mathrm{g} / \mathrm{kg} / \mathrm{min}$. Propofol was adjusted to keep the Narcotrend Index between $\mathrm{D}_{2}$ to $\mathrm{E}_{0}{ }^{[25]}$. The ventilation was adjusted to keep end tidal $\mathrm{CO}_{2}$ between 35 to $45 \mathrm{mmHg}$. All surgeries were performed by the one senior surgeon and same pneumoperitoneum apparatus to reduce surgical interference. During laparoscopy, $\mathrm{CO}_{2}$ was insufflated intraperitoneally to maintain an intra-abdominal pressure between 10 to $12 \mathrm{mmHg}$. All patients received tropisetron mesylate $6 \mathrm{mg}$ and fentanyl $2 \mu \mathrm{g} / \mathrm{kg}$ I.V. 20min before the end of the operation. Propofol and remifentanil were stopped when the trocars were removed. All surgical incisional wounds were infiltrated with $0.5 \%$ bupivacaine $10 \mathrm{ml}$ at the end of surgery.

In all groups, for various types of postoperative pain, intravenous tramadol $(1 \mathrm{mg} / \mathrm{kg})$ as a rescue analgesic was administrated when the patients requested in the recovery room and ward by the experienced nurses blinding to the investigation. The drugs would be repeatedly infused until patients felt unpainful and satisfactory.

After surgery, another experienced and independent doctor blinding to medication and grouping went to the ward and evaluated shoulder pain both at rest and with motion (side-lying, standing and walking) at $6 \mathrm{hr}, 24 \mathrm{hr}$ and $48 \mathrm{hr}$, respectively. The incidence and intensity of shoulder pain were assessed on a $10-\mathrm{cm}$ horizontal visual analog scale (VAS) with anchors "no shoulder pain (0)" and "worst possible pain (100)". The patient was considered to have PLSP if the VAS $>0$, either at rest or with motion, at any observing time point. In each group, the number of patients with VAS $=0$ at each time point was recorded to evaluate the overall incidence of PLSP. Meanwhile, the incisional pain was also assessed by similar methods.

At the end of the observation, the maximal shoulder pain (VAS) was assessed. The impact of shoulder pain on the patients' QOL was evaluated by modified Brief Pain Inventory (BPI) scales with four variables regarding to QOL (including activity, mood, walking ability and sleep), which was quantified ranging from no interference (0) to complete interference $(10)[26,27]$. The amount of rescue tramadol delivery for PLSP and the amount in whole duration were recorded. Meanwhile, the side effect profiles (nausea and vomiting, itch of skin, dizziness, and oversedation) and the severe complications associated with NSAIDs (e.g. cardiovascular events, stroke, coagulopathy and gastrointestinal diseases) were also assessed.

\section{Statistical analysis}

The overall incidence of PLSP was the primary outcome in the present study. The incidence of PLSP in the control group was set as $60 \%$ according to our pilot study. With reference to several similar previous reports $[5,6,8,19]$, either of our two regimens was expected to decrease the incidence by $50 \%$. Based on a method of sample size calculation [28], 40 patients for each group were required for a chi-square test with power $=0.8$ and $a=0.05$ (2-sided). Considering the possible $5 \%$ drop outs, we decided to recruit 126 patients. Demographic and surgical data were expressed as mean \pm standard deviation and number of patients. The incidence of pain and interference caused by shoulder pain were expressed as number of patients (percent). Because of a non-normal distribution, VAS and BPI scores were expressed as median and interquartile range. The primary outcome and other dichotomous outcomes were compared by $\chi^{2}$ test or Fisher's exact test, using the global $P$-value for significant. The treatment effect was the difference of incidence between each treatment (Group M or Group S) and the control (Group C), presented as rate difference (RD) with 95\% confidence interval (CI). Efficacy results were calculated by means of generalized linear models with a binomial distribution and identity link function. To control for type I error, the Bonferroni correction for multiple testing was applied to the $P$-values from pairwise comparisons between the treatment groups and the control. The maximal PLSP intensity was analyzed with Mann-Whitney U Test. All analyses were per-protocol and were computed using SAS 9.1 software (SAS Institute, Cary, NC, USA) and SPSS 15.0 software (SPSS, Chicago, IL, USA). All reported $P$-values are two-sided.

\section{Results}

From Jul 2011 to Oct 2011, a total of 126 patients were recruited into this study, 3 of those have been excluded because of being converted to laparotomy ( 2 patients) and being accidentally received other analgesics (1 patient). 123 patients (40 in the M group, 41 in the $S$ group and 42 in group $C$ ) were finally included in data analysis (Figure 1).

Demographic and surgical data were shown in Table 1. There were no clinically important differences regarding age, weight, height, main diagnosis, length of pneumoperitoneum, and total dose of analgesics during surgery between the three groups (Table 1). 


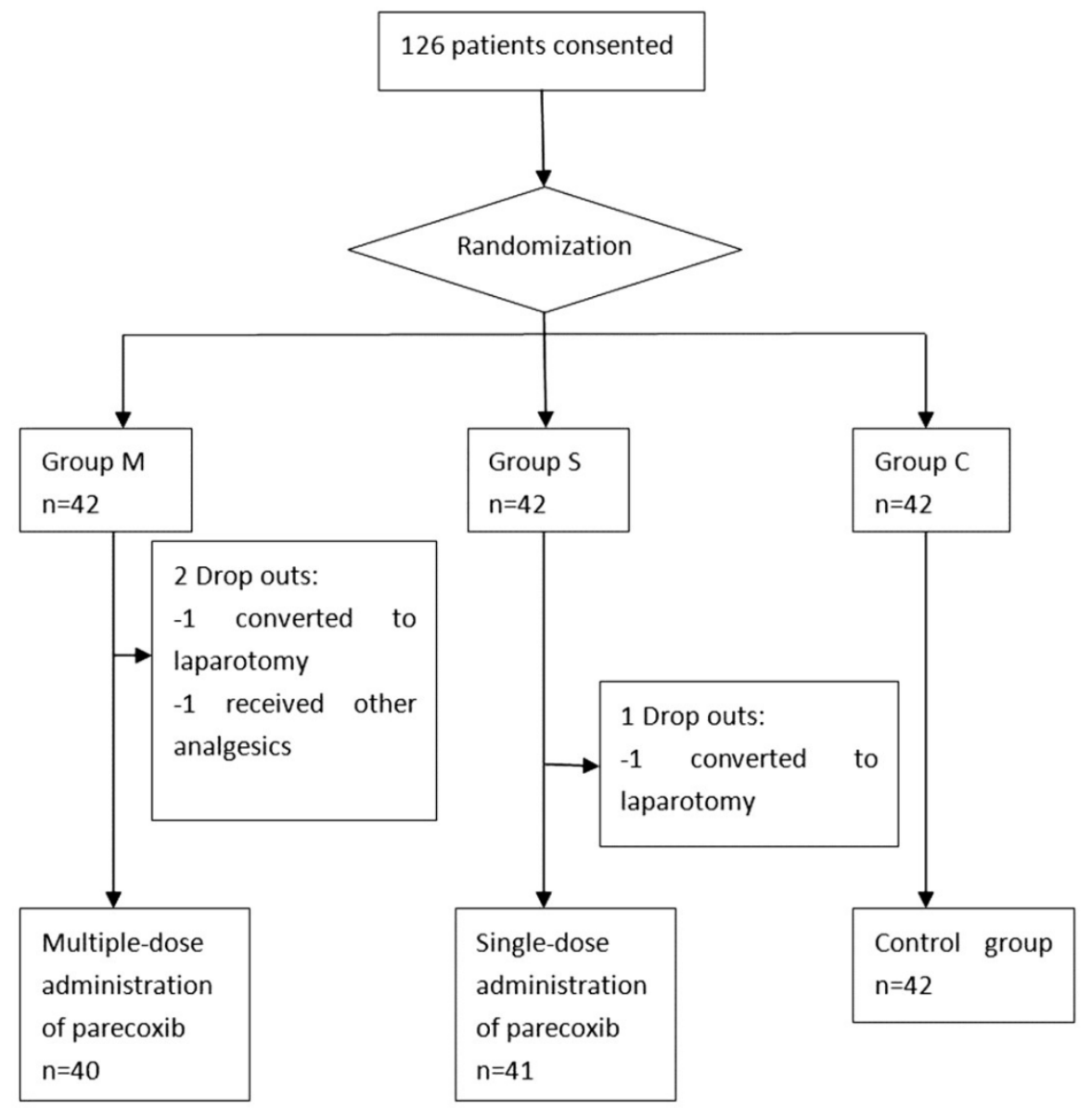

Figure I. Flowchart of randomization and group allocation

Table I. Demographic, surgical and analgesics data

\begin{tabular}{|c|c|c|c|}
\hline & $\begin{array}{l}\text { Group M } \\
n=40\end{array}$ & $\begin{array}{l}\text { Group S } \\
\mathrm{n}=41\end{array}$ & $\begin{array}{l}\text { Group C } \\
n=42\end{array}$ \\
\hline Age (yr) & $35.6 \pm 6.9$ & $34.6 \pm 6.7$ & $34.9 \pm 7.0$ \\
\hline Height (cm) & $159.3 \pm 4.8$ & $158.5 \pm 4.0$ & $157.8 \pm 3.9$ \\
\hline Weight $(\mathrm{kg})$ & $55.4 \pm 7.5$ & $52.5 \pm 7.0$ & $53.8 \pm 9.5$ \\
\hline ASA status (I/II) & $33 / 7$ & $36 / 5$ & $34 / 8$ \\
\hline \multicolumn{4}{|l|}{ Main diagnosis } \\
\hline Uterine fibroids & 14 & 13 & 17 \\
\hline Infertility & 18 & 15 & 14 \\
\hline Ovarian cyst & 7 & 10 & 7 \\
\hline Ectopic pregnancy & 1 & 2 & 2 \\
\hline Others & 0 & 1 & 2 \\
\hline $\begin{array}{l}\text { Length of pneumoperito- } \\
\text { neum (min) }\end{array}$ & $41.3 \pm 23.4$ & $40.9 \pm 23.5$ & $42.9 \pm 26.9$ \\
\hline \multicolumn{4}{|l|}{$\begin{array}{l}\text { Total doses of analgesics } \\
(\mu \mathrm{g})\end{array}$} \\
\hline Remifentanil & $838.0 \pm 401.4$ & $851.6 \pm 479.9$ & $833.8 \pm 482.4$ \\
\hline Fentanyl & $92.9 \pm 11.8$ & $94.5 \pm 11.9$ & $90.1 \pm 14.5$ \\
\hline
\end{tabular}

Group M: multiple-dose of parecoxib; Group S: single-dose of parecoxib; Group C: normal saline control:

Data were expressed as mean \pm standard deviation or number patients.
The data of shoulder pain was shown in Table 2. Overall, 66 of 123 (54\%) patients reported shoulder pain over the $48 \mathrm{hrs}$ assessment period. All these 66 patients experienced PLSP immediately after standing, and 24 of them $(7,11$ and 6 patients in group M, S and $C$, respectively) suffered from shoulder pain only after motion, but not at rest. The overall incidence of shoulder pain in the group C was $61.9 \%$. In group $\mathrm{M}$, the overall incidence of PLSP was decreased ( $\mathrm{RD}=-24.4 \%$; 95\% CI: 3.4 45.4\%; $\mathrm{P}=0.023$ ) in comparison to control group (Table 2). In contrast, the single-dose regimen of parecoxib showed no significant reduction for incidence of shoulder pain $(\mathrm{RD}=-0.9 \%$; 95\% CI: $-21.9 \sim 20.0 \%$; $\mathrm{P}=0.931$ ). During the study period, the maximal pain score of PLSP was significant lower $(p=0.004)$ in group $M$ than that in group C (Table 2). Meanwhile, the overall incidence of incisional pain were also lower in group $M$ than that in group $C$ ( $\mathrm{RD}=-20.7 \%$; 95\% CI: 2.5 38.9\%; $\mathrm{P}=0.026$ ). The detailed data of incisional pain was shown in Table 3.

As shown in Table 4, In comparison to group C, multiple-dose regimen decreased the impact of PLSP for activity (RD=-20.7\%; 95\% CI: 2.5 38.9\%; $\mathrm{P}=0.026$ ) 
and $\operatorname{mood}(\mathrm{RD}=-18.7 \%$; 95\% CI: $-34.3 \sim-3.1 \% ; \mathrm{P}=0.019)$ respectively. Nevertheless, for walking ability and sleep, there were no significant difference for each intervention group relative to control group.

The number of patients receiving rescue tramadol for postoperative pain were lower in group $\mathrm{M}$ than that in group C (Table 5).

The incidences of complications (nausea and vomiting, itch of skin, dizziness, and oversedation) during the study period were similar among the three groups. No severe complications were observed in this study.

Table 2. Incidence and intensity of postoperative shoulder pain

\begin{tabular}{|c|c|c|c|c|c|c|c|}
\hline & \multirow{2}{*}{$\begin{array}{l}\text { Group C } \\
n=42\end{array}$} & \multirow{2}{*}{$\begin{array}{l}\text { Group M } \\
\mathrm{n}=40\end{array}$} & \multicolumn{2}{|c|}{ Group M vs. Group C } & \multirow{2}{*}{$\begin{array}{l}\text { Group S } \\
\mathrm{n}=41\end{array}$} & \multicolumn{2}{|c|}{ Group S vs. Group C } \\
\hline & & & RD (95\% CI) & $P$ & & $\mathrm{RD}(95 \% \mathrm{CI})$ & $P$ \\
\hline \multicolumn{8}{|l|}{ Incidence } \\
\hline Overall & $26(61.9)$ & $15(37.5)$ & $-24.4(-45.4 \sim-3.4)$ & 0.026 & $25(61)$ & $-16.2(-35.1 \sim 2.7)$ & 0.931 \\
\hline \multicolumn{8}{|l|}{ Intensity } \\
\hline \multicolumn{8}{|l|}{ Rest } \\
\hline $6 h r$ & $0(0-0)$ & $0(0-0)$ & & & $0(0-0)$ & & \\
\hline mild & $2(4.8)$ & $1(2.5)$ & & & $1(2.4)$ & & \\
\hline medium & $3(7.1)$ & $2(5)$ & & & $3(7.3)$ & & \\
\hline severe & $1(2.4)$ & $0(0)$ & & & $1(2.4)$ & & \\
\hline $24 h r$ & $0(0-41.25)$ & $0(0-0)$ & & & $0(0-0)$ & & \\
\hline mild & $6(14.3)$ & $1(2.5)$ & & & $5(12.2)$ & & \\
\hline medium & $11(26.2)$ & $6(15)$ & & & $4(9.8)$ & & \\
\hline severe & $0(0)$ & $0(0)$ & & & $0(0)$ & & \\
\hline $48 h r$ & $0(0-0)$ & $0(0-0)$ & & & $0(0-0)$ & & \\
\hline mild & $1(2.4)$ & $4(10)$ & & & $2(4.9)$ & & \\
\hline medium & $3(7.1)$ & $0(0)$ & & & $2(4.9)$ & & \\
\hline severe & $2(4.8)$ & $0(0)$ & & & $1(2.4)$ & & \\
\hline \multicolumn{8}{|l|}{ Motion } \\
\hline $6 h r$ & $0(0-31)$ & $0(0-0)$ & & & $0(0-0)$ & & \\
\hline mild & $2(4.8)$ & $4(10)$ & & & $2(4.9)$ & & \\
\hline medium & $9(21.4)$ & $5(12.5)$ & & & $5(12.2)$ & & \\
\hline severe & $1(2.4)$ & $0(0)$ & & & $1(2.4)$ & & \\
\hline $24 h r$ & $26.5(0-50.75)$ & $0(0-43.75)$ & & & $20(0-40)$ & & \\
\hline mild & $6(14.3)$ & $2(5)$ & & & $10(24.4)$ & & \\
\hline medium & $18(42.9)$ & $10(25)$ & & & $11(26.8)$ & & \\
\hline severe & $0(0)$ & $1(2.5)$ & & & $3(7.3)$ & & \\
\hline $48 h r$ & $0(0-15)$ & $0(0-0)$ & & & $0(0-15)$ & & \\
\hline mild & $5(11.9)$ & $7(17.5)$ & & & $6(14.6)$ & & \\
\hline medium & $9(21.4)$ & $0(0)$ & & & $6(14.6)$ & & \\
\hline severe & $2(4.8)$ & $0(0)$ & & & $1(2.4)$ & & \\
\hline Maximal & $40(0-70)$ & $0(0-43.75)$ & & 0.004 & $30(0-50)$ & & 0.179 \\
\hline
\end{tabular}

Group M: multiple-dose of parecoxib; Group S: single-dose of parecoxib; Group C: normal saline control;

RD: rate difference; $\mathrm{CI}$ : confidence interval;

Data were expressed as number patients (percent) or median (interquartile range);

Mild: visual analog scale=1-30; medium: visual analog scale=31-70; severe: visual analog scale=71-100. 
Table 3. Incidence and intensity of postoperative incisional pain

\begin{tabular}{|c|c|c|c|c|c|c|c|}
\hline & \multirow{2}{*}{$\begin{array}{l}\text { Group C } \\
n=42\end{array}$} & \multirow{2}{*}{$\begin{array}{l}\text { Group M } \\
\mathrm{n}=40\end{array}$} & \multicolumn{2}{|c|}{ Group M vs. Group C } & \multirow{2}{*}{$\begin{array}{l}\text { Group S } \\
n=41\end{array}$} & \multicolumn{2}{|c|}{ Group S vs. Group C } \\
\hline & & & RD $(95 \%$ CI $)$ & $P$ & & RD (95\% CI) & $P$ \\
\hline \multicolumn{8}{|l|}{ Incidence } \\
\hline Overall & $26(61.9)$ & $15(37.5)$ & $-20.7(-38.9 \sim-2.5)$ & 0.026 & $25(61)$ & $-8.9(-28.7 \sim 11.0)$ & 0.380 \\
\hline \multicolumn{8}{|l|}{ Intensity } \\
\hline $6 h r$ & $0(0-24.75)$ & $0(0-0)$ & & & $0(0-0)$ & & \\
\hline mild & $5(11.9)$ & $5(12.5)$ & & & $1(2.4)$ & & \\
\hline medium & $6(14.3)$ & $0(0)$ & & & $4(9.8)$ & & \\
\hline severe & $2(4.8)$ & $0(0)$ & & & $0(0)$ & & \\
\hline $24 h r$ & $0(0-0)$ & $0(0-0)$ & & & $0(0-0)$ & & \\
\hline mild & $1(2.4)$ & $1(2.5)$ & & & $4(9.8)$ & & \\
\hline medium & $8(19.0)$ & $1(2.5)$ & & & $4(9.8)$ & & \\
\hline severe & $0(0)$ & $0(0)$ & & & $0(0)$ & & \\
\hline $48 h r$ & $0(0-0)$ & $0(0-0)$ & & & $0(0-0)$ & & \\
\hline mild & $3(7.1)$ & $2(5)$ & & & $1(2.4)$ & & \\
\hline medium & $2(4.8)$ & $0(0)$ & & & $1(2.4)$ & & \\
\hline severe & $0(0)$ & $0(0)$ & & & $0(0)$ & & \\
\hline
\end{tabular}

Group M: multiple-dose of parecoxib; Group S: single-dose of parecoxib; Group C: normal saline control;

$\mathrm{RD}$ : rate difference; $\mathrm{CI}$ : confidence interval;

Data were expressed as number patients (percent) or median (interquartile range);

Mild: visual analog scale=1-30; medium: visual analog scale=31-70; severe: visual analog scale=71-100.

Table 4. Impact of shoulder pain on recovery

\begin{tabular}{|c|c|c|c|c|c|c|c|}
\hline & Group C & Group M & Group M vs. Gro & & Group S & Group S vs. Group C & \\
\hline & $\mathrm{n}=42$ & $\mathrm{n}=40$ & RD (95\% CI) & $P$ & $\mathrm{n}=41$ & $\mathrm{RD}(95 \% \mathrm{CI})$ & $P$ \\
\hline \multicolumn{8}{|l|}{ Activity } \\
\hline Incidence & 15(35.7) & $6(15.0)$ & $-20.7(-38.9 \sim-2.5)$ & 0.026 & $8(19.5)$ & $-16.2(-35.1 \sim 2.7)$ & 0.066 \\
\hline BPI score & $0(0-5.25)$ & $0(0-0)$ & & & $0(0-0)$ & & \\
\hline mild & $0(0)$ & $2(5)$ & & & $1(2.4)$ & & \\
\hline medium & $7(16.7)$ & $2(5)$ & & & $4(9.8)$ & & \\
\hline severe & $8(19.0)$ & $2(5)$ & & & $3(7.3)$ & & \\
\hline \multicolumn{8}{|c|}{ Walking ability } \\
\hline Incidence & $1(2.4)$ & $2(5.0)$ & $2.6(-5.6 \sim 10.8)$ & 0.530 & $3(7.3)$ & $4.9(-4.3 \sim 14.1)$ & 0.293 \\
\hline BPI score & $0(0-0)$ & $0(0-0)$ & & & $0(0-0)$ & & \\
\hline mild & $1(2.4)$ & $0(0)$ & & & $1(2.4)$ & & \\
\hline medium & $0(0)$ & $2(5)$ & & & $1(2.4)$ & & \\
\hline severe & $0(0)$ & $0(0)$ & & & $1(2.4)$ & & \\
\hline \multicolumn{8}{|l|}{ Sleep } \\
\hline Incidence & $7(16.7)$ & $3(7.5)$ & $-9.2(-23.1 \sim 4.7)$ & 0.197 & $7(17.1)$ & $0.4(-15.7 \sim 16.5)$ & 0.961 \\
\hline BPI score & $0(0-0)$ & $0(0-0)$ & & & $0(0-0)$ & & \\
\hline mild & $2(4.8)$ & $1(2.5)$ & & & $0(0)$ & & \\
\hline medium & $1(2.4)$ & $1(2.5)$ & & & $5(12.2)$ & & \\
\hline severe & $4(9.5)$ & $1(2.5)$ & & & $2(4.9)$ & & \\
\hline \multicolumn{8}{|l|}{ Mood } \\
\hline Incidence & $11(26.2)$ & $3(7.5)$ & $-18.7(-34.3 \sim-3.1)$ & 0.019 & $7(17.1)$ & $-9.1(-26.7 \sim 8.4)$ & 0.310 \\
\hline BPI score & $0(0-3.125)$ & $0(0-0)$ & & & $0(0-0)$ & & \\
\hline mild & $1(2.4)$ & $0(0)$ & & & $1(2.4)$ & & \\
\hline medium & $6(14.3)$ & $2(5)$ & & & $5(12.2)$ & & \\
\hline severe & $4(9.5)$ & $1(2.5)$ & & & $1(2.4)$ & & \\
\hline
\end{tabular}

Group M: multiple-dose of parecoxib; Group S: single-dose of parecoxib; Group C: normal saline control;

RD: rate difference; CI: confidence interval; BPI: Brief Pain Inventory score;

Data were expressed as number patients (percent) or median (interquartile range);

Mild: $\mathrm{BPI}<3$; medium: $\mathrm{BPI}=3-7$; severe: $\mathrm{BPI}>7$. 
Table 5. The use of rescue analgesics

\begin{tabular}{|c|c|c|c|c|c|c|c|}
\hline & \multirow{2}{*}{$\begin{array}{l}\text { Group C } \\
n=42\end{array}$} & \multirow{2}{*}{$\begin{array}{l}\text { Group M } \\
\mathrm{n}=40\end{array}$} & \multicolumn{2}{|c|}{ Group M vs. Group C } & \multirow{2}{*}{$\begin{array}{l}\text { Group S } \\
\mathrm{n}=41\end{array}$} & \multicolumn{2}{|c|}{ Group S vs. Group C } \\
\hline & & & $\mathrm{RD}(95 \% \mathrm{CI})$ & $P$ & & RD $(95 \% \mathrm{CI})$ & $P$ \\
\hline \multicolumn{8}{|l|}{ For shoulder pain } \\
\hline The number of patients & $13(31.0)$ & $3(7.5)$ & $-23.5(-39.6 \sim-7.3)$ & 0.005 & $7(17.1)$ & $-13.9(-32.0 \sim 4.2)$ & 0.133 \\
\hline \multicolumn{8}{|l|}{ The amount of tramadol } \\
\hline 1 time & $11(26.2)$ & $1(2.5)$ & & & $5(12.2)$ & & \\
\hline 2 times & $2(4.8)$ & $2(5)$ & & & $2(4.9)$ & & \\
\hline \multicolumn{8}{|l|}{ For total pain } \\
\hline The number of patients & $25(59.5)$ & $10(25)$ & $-34.5(-54.5 \sim-14.5)$ & 0.001 & $17(41.5)$ & $-18.1(-39.2 \sim 3.1)$ & 0.094 \\
\hline \multicolumn{8}{|l|}{ The amount of tramadol } \\
\hline 1 time & $14(33.3)$ & $7(17.5)$ & & & $10(24.4)$ & & \\
\hline 2 times & $8(19)$ & $2(5)$ & & & $4(9.8)$ & & \\
\hline 3 times & $2(4.8)$ & $1(2.5)$ & & & $2(4.9)$ & & \\
\hline 4 times & $1(2.4)$ & $0(0)$ & & & $1(2.4)$ & & \\
\hline
\end{tabular}

Group M: multiple-dose of parecoxib; Group S: single-dose of parecoxib; Group C: normal saline control;

RD: rate difference; CI: confidence interval;

Data were expressed as number patients (percent).

\section{Discussion}

Laparoscopic surgical procedures belong to those with the highest incidence of moderate to severe pain for $24 \mathrm{hr}$ postoperatively [29]. Alanoglu et al. suggested that selective COX-2 inhibitor was an ideal analgesic drug after laparoscopic surgery [15]. To date, however, appropriate dosage and timing of parecoxib are still not clear. Akaraviputh et al. indicated that preoperative infusion $20 \mathrm{mg}$ parecoxib could reduce the postoperative opioid consumption [30], and $\mathrm{Pa}$ padima et al. showed that preoperative parecoxib $40 \mathrm{mg}$ was efficacious for pain control and opioids sparing following laparoscopic cholecystectomy [31]. In another study, parecoxib $40 \mathrm{mg}$ twice daily attenuated worst pain in laparoscopic cholecystectomy ${ }^{[32]}$, but a latest study found that the efficacy of same medication on piritramide consumption and pain relief was weak [33]. For gynecologic laparoscopies, the results of parecoxib are also contradictory [34,35]. Therefore, investigation of an appropriate dosing regimen of parecoxib for postlaparoscopic pain, especially for shoulder pain, is of clinical importance.

A recent study in Thailand reported that preemptive parecoxib 40mg reduced PLSP intensity within postoperative $24 \mathrm{hrs}$ [35]. However, the current results showed that single-dose parecoxib had no significant superiority over shoulder pain control. We speculated that two factors could be involved in this difference. First, in the present study, shoulder pain was assessed not only at rest but also with motion. Phelps et al. indicated that standing could lead to shoulder pain after gynecologic laparoscopy [5]. Similarly, our study also showed that motion was closely related to the occurrence of shoulder pain: all the 66 patients with PLSP felt pain soon after standing, and 24 of them experienced shoulder pain only after movement. The single-dose regimen probably could not alleviate shoulder pain induced by motion, and then did not reduce the overall incidence of PLSP. Second, we evaluated PLSP during postoperative 48 hrs due to PLSP usually occurs after postoperative $6 \mathrm{hr}$ [36] and lasts $48-72 \mathrm{hrs}$ [7]. Because the estimated terminal half-life of valdecoxib, the active moiety of parecoxib, is about 7hrs [37], single administration of parecoxib may not provide $48 \mathrm{hrs}$ pain relief. Whereas, in group $M$, although the effect of parecoxib was just kept about 30hrs after surgery through three-dose regimen, the overall incidence of PLSP was reduced by $40 \%$. The results indicated that COX inhibition must be maintained an enough time after surgery to inhibit the occurrence of shoulder pain. For the clinicians, our important finding clearly suggested that adequate follow-up treatment of COX-2 inhibitor was necessary to relieve PLSP. In several countries, postoperative intravenous analgesia is infrequently used because the majority of patients are not admitted as in-patients following elective gynecologic laparoscopy. In such cases, intravenous followed by oral COX-2 specific inhibitors (e.g. celecoxib, valdecoxib) may be the favorable alternatives.

It is well known that postoperative surgical pain affects patients' recovery $[38,39]$. However, to the best of our knowledge, no study ever investigated the impact of PLSP on postoperative recovery. In this study, modified BPI score, a widely used measurement tool for assessing clinical pain, was applied to measure how much shoulder pain had interfered with patients' 
performance after laparoscopy. Our results demonstrated that 15 of $42(35.7 \%)$ patients described PLSP interfered their general activities, and 8 patients $(19.0 \%)$ estimated this interference as "Severe" (score>7) in group C. Early resumption of activities of daily living is the main advantage of laparoscopic surgery over open surgery [40,41], but several patients had to decrease early out-of-bed activities due to PLSP induced by these activities. And multiple-dose regimen of parecoxib might relieve the impact of PLSP for general activities mainly by reducing the occurrence of shoulder pain caused by various motions.

Regrettably, parecoxib and other NSAIDs were banned in United States, Australia and other states mainly due to the risk of adverse cardiovascular effects. However, several studies have reported that short-term use (< 1 week) of COX-2 inhibitors improved pain management without causing serious complications after non-cardiac surgery procedures [42]. It has been reported that, for the patients with no more than moderate cardiovascular risk factors, parenteral parecoxib for 3 days following oral valdecoxib for 7 days improved the pain relief and did not increase morbidity or mortality in major general, orthopedic, and gynecological procedures [43]. Thus, the clinicians shall be familiar with the adverse effects of the drug to avoid the potential risks.

It must be pointed out that off-label use of parecoxib was applied in group M. Parecoxib $40 \mathrm{mg}$ twice a day given is the maximum dosage recommended by the manufacturer for I.V. application in adults. In the present study, considering parecoxib's analgesic duration and patients' rest time, the third infusion was delivered at approximate $20.5 \mathrm{hr}$ after initial dose. However, because our dosing interval was longer than the half-life of parecoxib and the total dose used for each patient was small, our three-dose regimen did not increase the occurrence of postoperative complications. Mehlisch et al. showed that single-dose of parecoxib 100mg appeared to be safe and well tolerated in oral surgery patients [44]. Furthermore, it has been reported that there are no dose-dependent effects on adverse events and no severe complications at doses up to parecoxib 200mg by intravenous injection in volunteers [45]. Indeed, we suggest that this simple and non-opioid multiple-dose regimen is an appropriate technique for pain management after gynecologic laparoscopy.

There were several possible limitations in this study. Firstly, we did not employ an "active" comparator drug as control in the present study. Comparison of parecoxib and other NSAIDs (e.g. celecoxib, ibuprofen) might help the clinicians to better un- derstand the specific role of parecoxib in PLSP and develop a preferable analgesic regimen following laparoscopic surgery. Secondly, we just performed assessment within postoperative $48 \mathrm{hrs}$ because most of the patients discharged at postoperative day 3. A prolonged assessment is necessary to clearly know whether suppression of shoulder pain continues after parecoxib is completely metabolized and eliminated. Thirdly, in our study, the rescue tramadol for other types of postoperative pain might interfere the assessment of shoulder pain. However, a previous study ever reported that intravenous tramadol 100mg did not influence the incidence of shoulder pain after laparoscopic cholecystectomy [46]. Finally, because of the implement of multiple pairwise treatment group comparisons in statistical analysis, the sample size calculation should be adjusted to increase sample sizes to preserve more reasonable power.

In conclusion, after gynecologic laparoscopy, multiple-dose, rather than single-dose, administration of parecoxib simultaneously decreased the incidence and intensity of shoulder pain and incisional pain, and alleviated the negative influence of shoulder pain on patients' recovery. In addition, this multiple-dose regimen did not enhance side effects. Therefore, for PLSP, multiple-dose administration of parecoxib may be an effective, safe and simple treatment to be implemented in clinical practice.

\section{Acknowledgments}

Special thanks to Prof Hongzhe Xie, an excellent gynecologist at Gynecologic Laparoscopic Medical Center of The First Affiliated Hospital of Sun Yat-sen University, for his substantial help and advice about the details of the surgical technique. Special thanks to Prof Aihua Lin and Dr Guanrong Zhang, two outstanding experts at Department of Medical Statistics of Sun Yat-sen University, for their professional help and advice about the statistical analysis.

\section{Competing Interests}

The authors have declared that no competing interest exists.

\section{References}

1. Chapron C, Fauconnier A, Goffinet F, Bréart G, Dubuisson JB. Laparoscopic surgery is not inherently dangerous for patients presenting with benign gynaecologic pathology. Results of a meta-analysis. Hum Reprod 2002;17:1334-42.

2. Harrell AG, Heniford BT. Minimally invasive abdominal surgery: lux et veritas past, present, and future. Am J Surg 2005;190:239-43.

3. Bijen CB, Vermeulen KM, Mourits MJ, de Bock GH. Costs and effects of abdominal versus laparoscopic hysterectomy: systematic review of controlled trials. PLoS One 2009;4:e7340.

4. Compeau C, McLeod NT, Ternamian A. Laparoscopic entry: a review of Canadian general surgical practice. Can J Surg 2011;54:315-20. 
5. Phelps P, Cakmakkaya OS, Apfel CC, Radke OC. A simple clinical maneuver to reduce laparoscopy-induced shoulder pain: a randomized controlled trial. Obstet Gynecol 2008;111:1155-60.

6. Chang SH, Lee HW, Kim HK, Kim SH, Kim DK. An evaluation of perioperative pregabalin for prevention and attenuation of postoperative shoulder pain after laparoscopic cholecystectomy. Anesth Analg 2009;109:1284-6.

7. Dixon JB, Reuben Y, Halket C, O'Brien PE. Shoulder pain is a common problem following laparoscopic adjustable gastric band surgery. Obes Surg 2005;15:1111-7.

8. Shin HY, Kim SH, Lee YJ, Kim DK. The effect of mechanical ventilation tidal volume during pneumoperitoneum on shoulder pain after a laparoscopic appendectomy. Surg Endosc 2010;24:2002-7.

9. Sarli L, Costi R, Sansebastiano G, Trivelli M, Roncoroni L. Prospective randomized trial of low-pressure pneumoperitoneum for reduction of shoulder-tip pain following laparoscopy. Br J Surg 2000;87:1161-5.

10. Koivusalo AM, Kellokumpu I, Lindgren L. Gasless laparoscopic cholecystectomy: comparison of postoperative recovery with conventional technique. Br J Anaesth 1996;77:576-80.

11. Vezakis A, Davides D, Gibson JS, Moore MR, Shah H, Larvin M, McMahon MJ. Randomized comparison between low-pressure laparoscopic cholecystectomy and gasless laparoscopic cholecystectomy. Surg Endosc 1999;13:890-3.

12. Narchi P, Benhamou D, Fernandez H. Intraperitoneal local anaesthetic for shoulder pain after day-case laparoscopy. Lancet 1991;338:1569-70.

13. Ng A, Swami A, Smith G, Robertson G, Lloyd DM. Is intraperitoneal levobupivacaine with epinephrine useful for analgesia following laparoscopic cholecystectomy? A randomized controlled trial. Eur J Anaesthesiol 2004;21:653-7.

14. Alexander JI. Pain after laparoscopy. Br J Anaesth 1997;79:369-78.

15. Alanoglu Z, Ateş Y, Orbey BC, Türkçapar AG. Preoperative use of selective COX-II inhibitors for pain management in laparoscopic nissen fundoplication. Surg Endosc 2005;19:1182-7.

16. Crocker S, Paech M. Preoperative rectal indomethacin for analgesia after laparoscopic sterilisation. Anaesth Intensive Care 1992;20:337-40.

17. Van EE R, Hemrika DJ, van der Linden CT. Pain relief following day-case diagnostic hysteroscopy-laparoscopy for infertility: a double-blind randomized trial with preoperative naproxen versus placebo. Obstet Gynecol 1993;82:951-4.

18. Edwards ND, Barclay K, Catling SJ, Martin DG, Morgan RH. Day case laparoscopy: a survey of postoperative pain and an assessment of the value of diclofenac. Anaesthesia 1991;46:1077-80.

19. Sandhu T, Paiboonworachat S, Ko-iam W. Effects of preemptive analgesia in laparoscopic cholecystectomy: a double-blind randomized controlled trial. Surg Endosc 2011;25:23-7.

20. Jabbour-Khoury SI, Dabbous AS, Gerges FJ, Azar MS, Ayoub CM, Khoury GS. Intraperitoneal and intravenous routes for pain relief in laparoscopic cholecystectomy. JSLS 2005;9:316-21.

21. Phinchantra P, Bunyavehchevin S, Suwajanakorn S, Wisawasukmongchol $\mathrm{W}$. The preemptive analgesic effect of celecoxib for day-case diagnostic laparoscopy. J Med Assoc Thai 2004;87:283-8.

22. Dembo G, Park SB, Kharasch ED. Central nervous system concentrations of cyclooxygenase-2 inhibitors in humans. Anesthesiology 2005;102:409-15.

23. Mehta V, Johnston A, Cheung R, Bello A, Langford RM. Intravenous parecoxib rapidly leads to COX-2 inhibitory concentration of valdecoxib in the central nervous system. Clin Pharmacol Ther 2008;83:430-5.

24. Koppert W, Wehrfritz A, Körber N, Sittl R, Albrecht S, Schüttler J, Schmelz M. The cyclooxygenase isozyme inhibitors parecoxib and paracetamol reduce central hyperalgesia in humans. Pain 2004;108:148-53.

25. Rundshagen I, Hardt T, Cortina K, Pragst F, Fritzsche T, Spies C. Narcotrend-assisted propofol/remifentanil anaesthesia vs clinical practice: does it make a difference? Br J Anaesth 2007;99:686-93.

26. Beauregard L, Pomp A, Choinière M. Severity and impact of pain after day-surgery. Can J Anaesth 1998;45:304-11.

27. Mendoza TR, Chen C, Brugger A, Hubbard R, Snabes M, Palmer SN, Zhang Q, Cleeland CS. Lessons learned from a multiple-dose post-operative analgesic trial. Pain 2004;109:103-9.

28. Whitley E, Ball J. Statistics review 4: sample size calculations. Crit Care 2002;6:335-41.

29. McGrath B, Elgendy H, Chung F, Kamming D, Curti B, King S. Thirty percent of patients have moderate to severe pain $24 \mathrm{hr}$ after ambulatory surgery: a survey of 5,703 patients. Can J Anaesth 2004;51:886-91.

30. Akaraviputh $\mathrm{T}$, Leelouhapong $\mathrm{C}$, Lohsiriwat V, Aroonpruksakul S. Efficacy of perioperative parecoxib injection on postoperative pain relief after laparoscopic cholecystectomy: a prospective, randomized study. World J Gastroenterol 2009;15:2005-8.
31. Papadima A, Lagoudianakis EE, Antonakis PT, Pattas M, Kremastinou F, Katergiannakis V, Manouras A, Georgiou L. Parecoxib vs. lornoxicam in the treatment of postoperative pain after laparoscopic cholecystectomy: a prospective randomized placebo-controlled trial. Eur J Anaesthesiol 2007;24:154-8.

32. Puolakka PA, Puura AI, Pirhonen RA, Ranta AU, Autio V, Lindgren L, Rorarius MG. Lack of analgesic effect of parecoxib following laparoscopic cholecystectomy. Acta Anaesthesiol Scand 2006;50:1027-32.

33. Abdulla S, Eckhardt R, Netter U, Abdulla W. A randomized, double-blind, controlled trial on non-opioid analgesics and opioid consumption for postoperative pain relief after laparoscopic cholecystectomy. Acta Anaesthesiol Belg 2012;63:43-50.

34. Ratchanon S, Phaloprakarn C, Traipak K. Pain control in laparoscopic gynecologic surgery with/without preoperative (preemptive) parecoxib sodium injection: a randomized study. J Med Assoc Thai 2011;94:1164-8.

35. Bunyavejchevin S, Prayoonwech C, Sriprajittichai P. Preemptive Analgesic Efficacy of Parecoxib vs Placebo in Infertile Women Undergoing Diagnostic Laparoscopy: Randomized Controlled Trial. J Minim Invasive Gynecol 2012; [Epub ahead of print].

36. Schulte-Steinberg H, Weninger E, Jokisch D, Hofstetter B, Misera A, Lange $\mathrm{V}$, Stein C. Intraperitoneal versus interpleural morphine or bupivacaine for pain after laparoscopic cholecystectomy. Anesthesiology 1995;82:634-40.

37. Yuan JJ, Yang DC, Zhang JY, Bible R Jr, Karim A, Findlay JW. Disposition of a specific cyclooxygenase-2 inhibitor, valdecoxib, in human. Drug Metab Dispos 2002;30:1013-21.

38. Joshi GP. Multimodal analgesia techniques and postoperative rehabilitation. Anesthesiol Clin North America 2005;23:185-202

39. Wei B, Qi CL, Chen TF, Zheng ZH, Huang JL, Hu BG, Wei HB. Laparoscopic versus open appendectomy for acute appendicitis: a meta analysis. Surg Endosc 2011;25:1199-208.

40. Basse L, Jakobsen DH, Bardram L, Billesbølle P, Lund C, Mogensen T, Rosenberg J, Kehlet H. Functional recovery after open versus laparoscopic colonic resection: a randomized, blinded study. Ann Surg 2005;241:416-23.

41. Zheng MH, Feng B, Lu AG, Li JW, Wang ML, Mao ZH, Hu YY, Dong F, Hu WG, Li DH, Zang L, Peng YF, Yu BM. Laparoscopic versus open right hemicolectomy with curative intent for colon carcinoma. World J Gastroenterol 2005;11:323-6.

42. White PF, Sacan O, Tufanogullari B, Eng M, Nuangchamnong N, Ogunnaike B. Effect of short-term postoperative celecoxib administration on patient outcome after outpatient laparoscopic surgery. Can J Anaesth 2007;54:342-8.

43. Nussmeier NA, Whelton AA, Brown MT, Joshi GP, Langford RM, Singla NK, Boye ME, Verburg KM. Safety and efficacy of the cyclooxygenase-2 inhibitors parecoxib and valdecoxib after noncardiac surgery. Anesthesiology 2006;104:518-26.

44. Mehlisch DR, Desjardins PJ, Daniels S, Hubbard RC. Single doses of parecoxib sodium intravenously are as effective as ketorolac in reducing pain after oral surgery. J Oral Maxillofac Surg 2003;61:1030-7.

45. Karim A, Laurent A, Qian J, Kuss M, Hubbard R. Single dose tolerability and pharmacokinetics of parecoxib sodium, a COX-2 specific inhibitor, following intravenous administration. San Francisco, California: Proceedings of the 2000 American Society of Anesthesiologists Annual Meeting, October 14-18, 2000. 2000:A-945.

46. Akinci SB, Ayhan B, Aycan IO, Tirnaksiz B, Basgul E, Abbasoglu O, Aypar U, Sayek I. The postoperative analgesic efficacy of intraperitoneal tramadol compared to normal saline or intravenous tramadol in laparoscopic cholecystectomy. Eur J Anaesthesiol 2008;25:375-81. 